\title{
The Scintillator Fiber Tracker and Muon Trigger at $\mathrm{D} \emptyset$
}

\author{
Maris Abolins ${ }^{* \dagger}$ \\ Department of Physics and Astronomy \\ Michigan State University \\ East Lansing, MI 48824-1116 \\ E-mail: iabōins@msu.èü
}

Abstract: The DØ experiment, now being commissioned at the Fermilab protonantiproton collider was recently upgraded. Among the most important changes have been the installation of a scintillating fiber tracker and a new muon detector with its associated trigger system. The salient features and performance of these devices is discussed and their current status and future prospects reviewed.

\section{Introduction}

Prior to starting Run IIA in March, 2001, the D $\varnothing$ detector underwent an extensive upgrade to prepare it for the new running environment with its higher instantaneous luminosity and decreased inter-bunch spacings and to improve its discovery potential for the possible new physics that would reveal itself. The main features of the upgrade, shown in Figure $1 \overline{1}$, include a solenoidal magnetic field, a silicon vertex detector, a new trigger system, a new muon detector system and a new scintillating fiber tracker. This paper describes features of two of these systems, the muon trigger and the scintillating fiber tracker.

\section{The Muon Trigger}

The Muon detector has undergone extensive changes for Run II. In Run I the active detector elements consisted exclusively of drift tubes. For Run II many of these have been retained being augmented by others of higher segmentation near the beam pipe. In addition for Run II we have added arrays of fast scintillators shown in Figure 2 . In the new, high luminosity environment with $132 \mathrm{~ns}$ bunch spacing, the fast timing possible with scintillators is an essential ingredient in triggering and background rejection. The $\mathrm{D} \varnothing$ trigger system consists

${ }^{*}$ Speaker.

${ }^{\dagger}$ For the D $\varnothing$ Collaboration. 


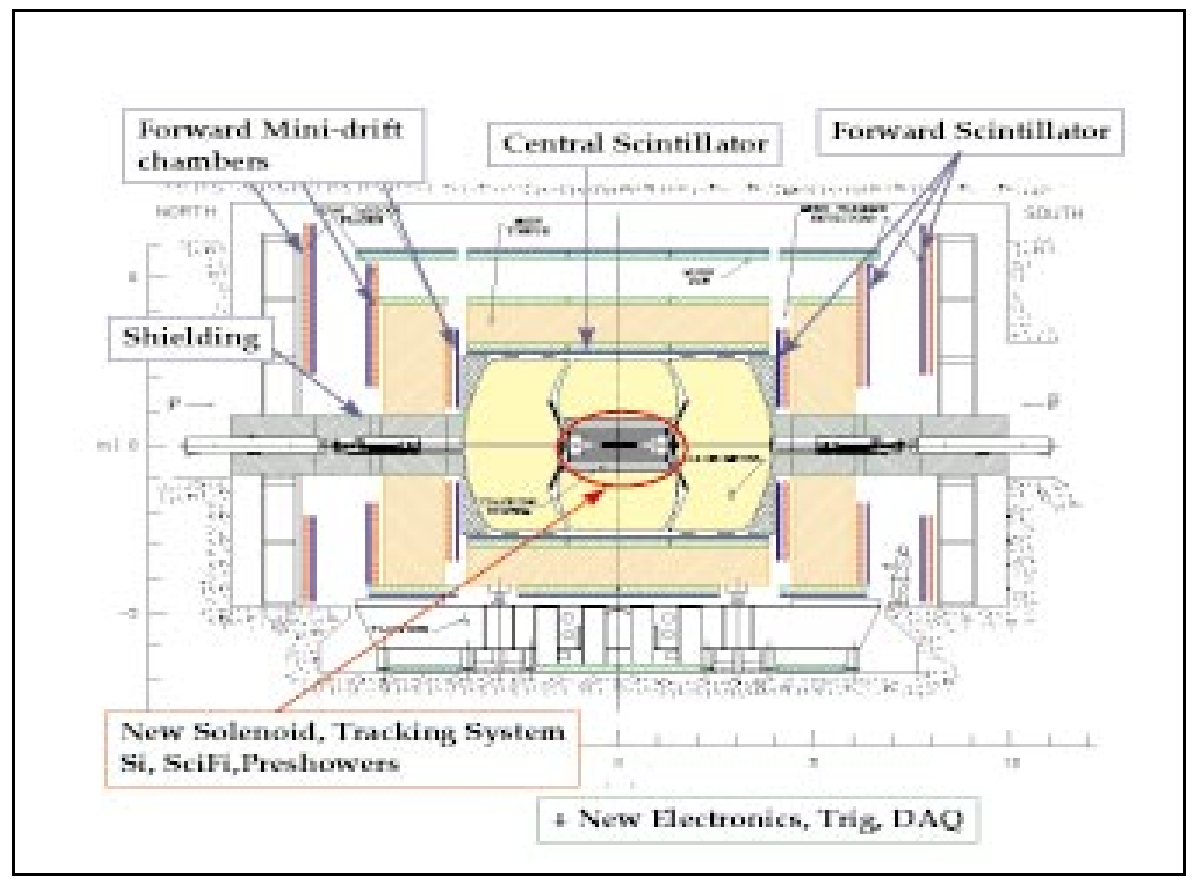

Figure 1: The DØ Detector Showing Main Upgrade Features.

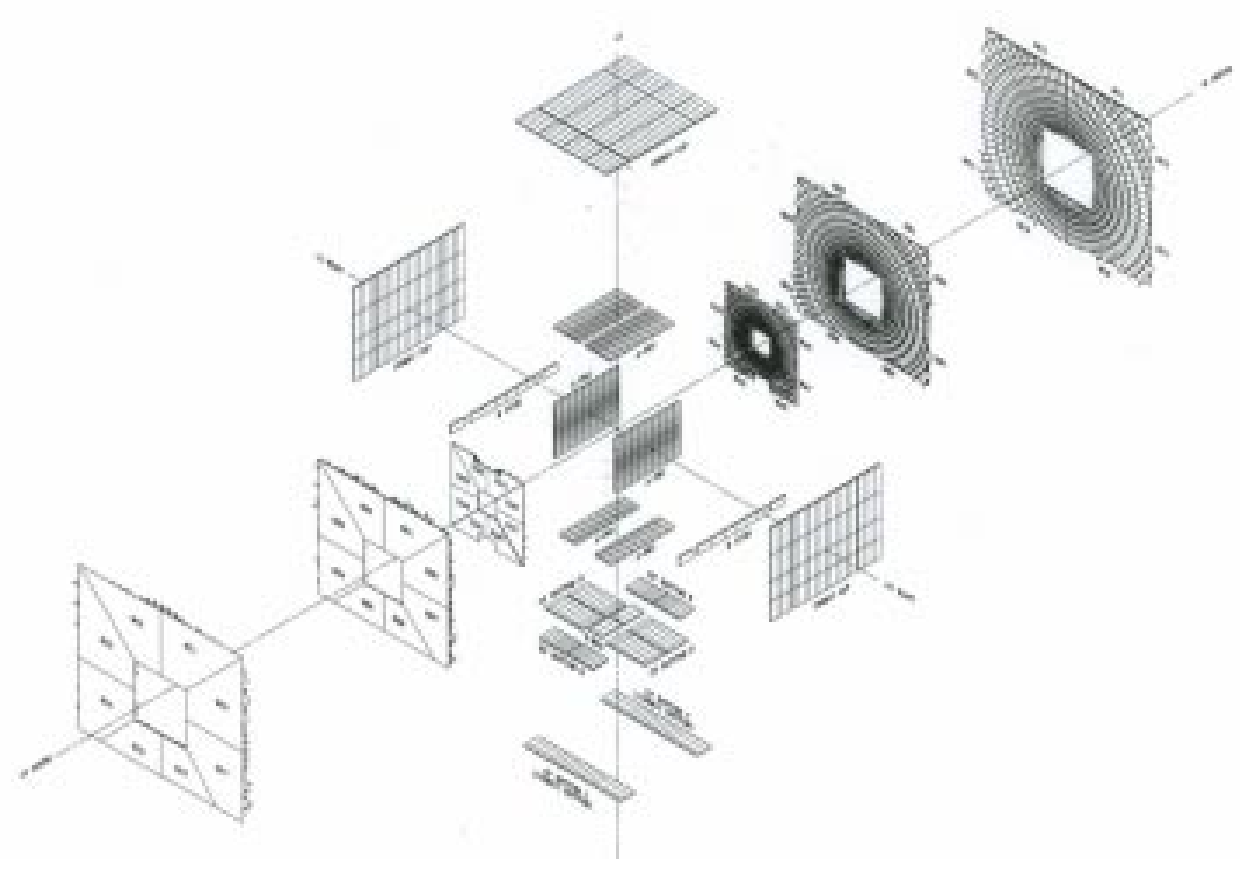

Figure 2: The Muon Scintillators

of three levels, level 1 made of custom fast electronics with an accept rate of $5 \mathrm{kHz}$, followed by level 2 with its specialized general purpose processors accepting $2 \mathrm{kHz}$ feeding level 3 , a farm of general purpose processors. The muon system differs from this arrangement in 
that it interposes an additional layer between levels 1 and 2 a level 1.5 whose function is to construct muon track segments for transmission to level 2. This is shown schematically in Figure in $_{i}^{-}$The Second Level Input Computer (SLIC), shown in Figure $\bar{A}_{0}^{-}$takes as

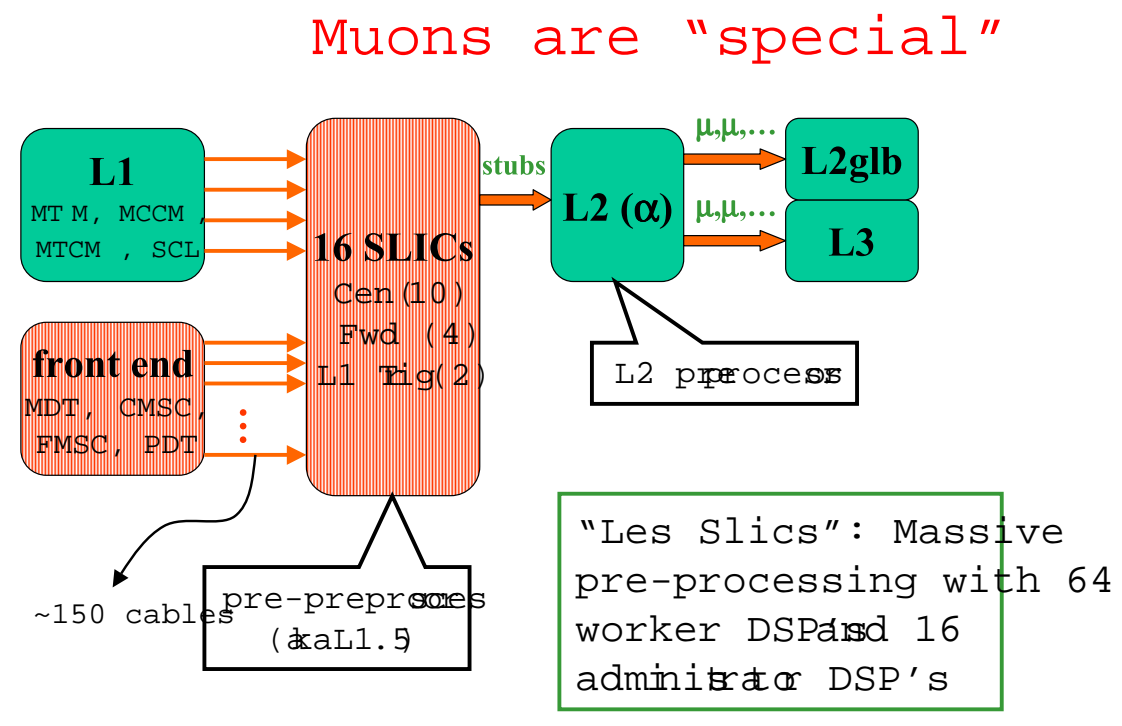

Figure 3: Muon Trigger System.

\section{The SLIC: one out of 16}

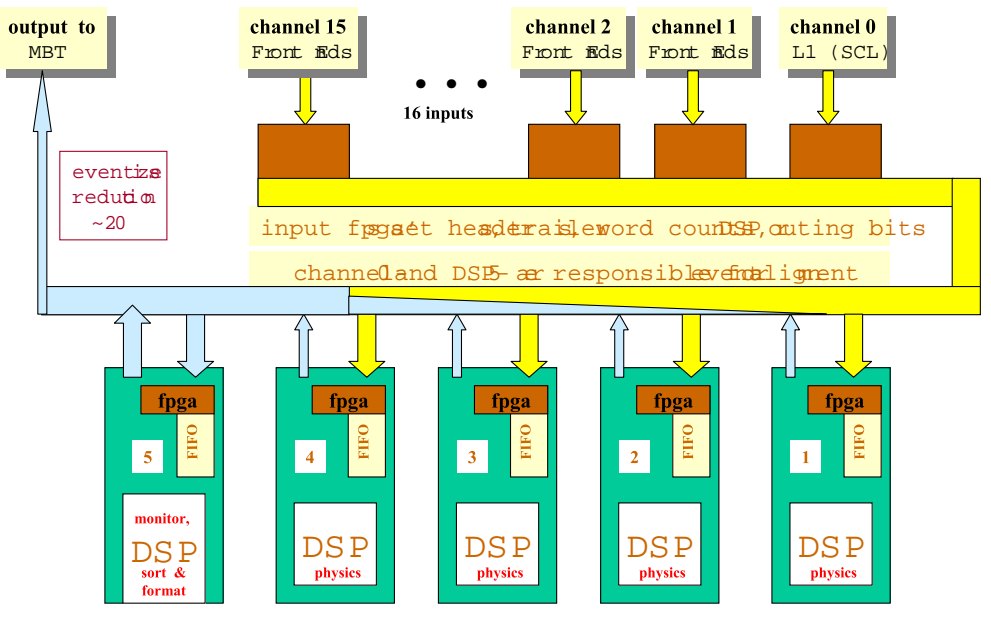

Figure 4: Schematic of a SLIC.

inputs the level 1 trigger information along with signals from the muon scintillators and drift tubes, computes all possible muon front-end stubs and transmits this information to one of the local level $2 \alpha$ local processors for more detailed analysis.

The results are then sent to the level 2 global processor to be combined with other local results in forming the level 2 decision. As of this writing, all elements of the muon trigger system are in place undergoing checkout and commissioning. 


\section{The Fiber Tracker}

Perhaps the most novel piece of the $\mathrm{D} \varnothing$ detector is the scintillating fiber tracker shown in cross-section in Figure long $835 \mu \mathrm{m}$ fibers arranged in one axial and one $\mathrm{u}, \mathrm{v}$ stereo doublet ( $3^{\circ}$ stereo angle).

There are a total of 77,000 individual fibers each connected to a $7-11 \mathrm{~m}$ long clear fiber that tranmits the signal to the optical detector, the Visual Light Photon Counter (VLPC). The VLPC is a solid state device, developed by Rockwell [i] $\left[\overline{2}\left[\begin{array}{l}2 \\ 2\end{array}\right]\right.$, with very high quantum efficiency $(80 \%$ in the visible) and superb energy resolution that is well suited for

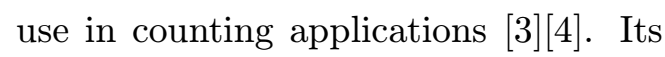
operation can be understood by considering the cartoon in Figure $\overline{6}_{i}^{1}$ A photon is converted in the intrinsic region, creating an electron-hole pair. The hole drifts into the drift region, where it knocks an electron out from an atom. The electron accelerates back through the gain region, knocking electrons from atoms

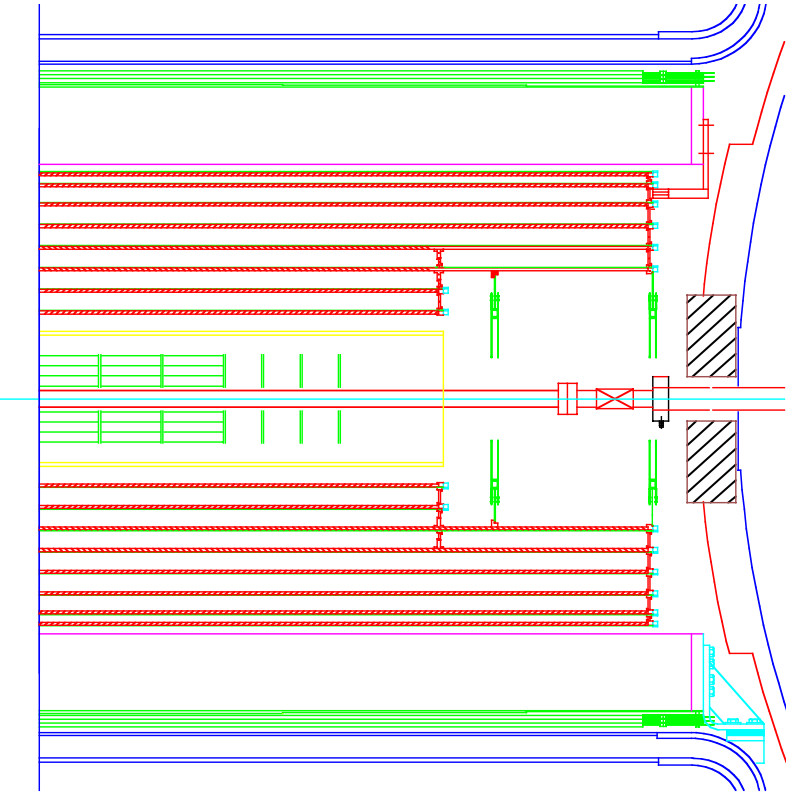

Figure 5: The scintillating fiber tracker. as it goes. The spacer region and substrate are for mechanical support and field shaping. Thus each photon generates a pulse of many electrons with gains of 20,000 to 60,000 achievable. The energy resolution of the device can be apreciated fom Figure itis which

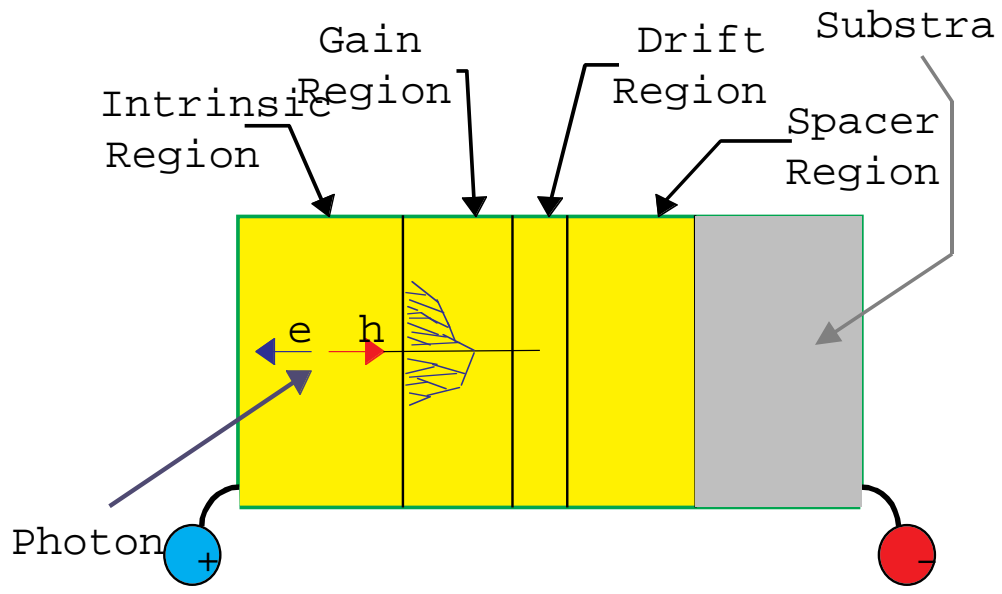

Figure 6: Photon Conversion in a VLPC.

shows the response of the device to individual photons. 


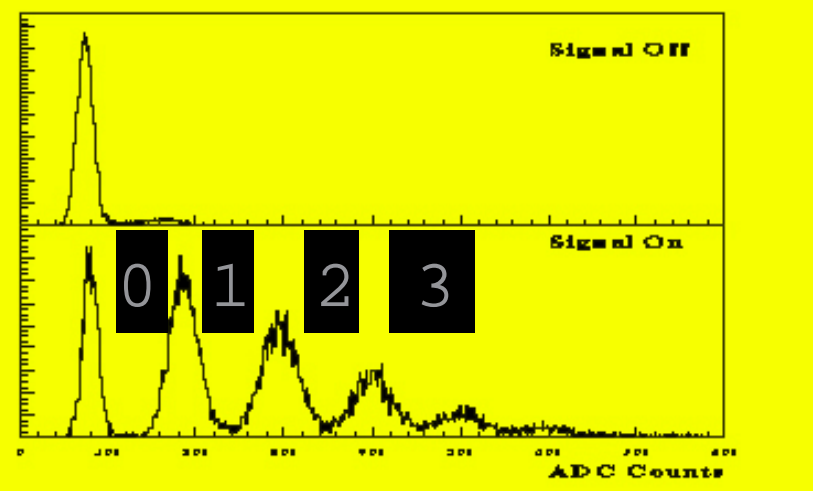

Figure 7: VLPC response to no photons (top) and 0, 1, 2 and 3 photons (bottom).

A potential inconvenience to the use of VLPC's is the need to run them at $7 \mathrm{~K}$. This problem has, however, been overcome and the VLPC's are operating in the DØ environment with sufficient efficiency to permit requiring 8 out of 8 layers in the trigger. Scintillating fiber tracking with VLPC readout represents an attractive solution to tracking in a high luminosity collider environment. The intrinsic speed and digital nature of the device makes it highly suitable for triggering in a high rate environment. Once installed the device is very stable and reliable. At the present time it is undergoing commissioning and we expect it to be fully operational later this fall when the full complement of readout electronics is installed.

\section{Acknowledgements}

I would like to thank Don Lincoln and Ken Johns for their help in assembling the materials for this talk. This work was supported by the National Science Foundation under grant PHY-9875000.

\section{References}

[1] M.D.Petroff, M.G.Stapelbroek and W.A.Kleinhans, Appl. Phys. Lett. 51 (1987) 406;

[2] M.D.Petroff and M.G.Stapelbroek, IEEE Trans. Nucl. Sci. NS-36 (1989) 158;

[3] M.D.Petroff and M.Atac, ibid., p.163;

[4] M.Atac et al., Nucl. Instr. and Meth. A314 (1992) 56. 